\title{
Comparison of Heart Rate Variability according to Performance in Elite Female Judo Athletes
}

\author{
Moon-jung Bae, MD・ Hyun-Chul Kim, PT, $\mathrm{PhD}^{\dagger} \cdot \mathrm{Ki}-J u n$ Park, PT, $\mathrm{PhD}^{1}$ \\ Department of Medicine and Science, Korean Sport \& Olympic Committee \\ ${ }^{1}$ Department of Physical Therapy, GangneungYeongdong University
}

Received: October 30, 2019 / Revised: November 29, 2019 / Accepted: December 17, 2019

(C) 2020 J Korean Soc Phys Med

\section{| Abstract |}

PURPOSE: This study was to investigate examined the differences in the autonomic nervous functions of elite female judo athletes according to their performance by measuring the heart rate variability (HRV).

METHODS: Sixteen elite female judo players participated in this study. The participants were divided into a high performance and low performance group according to the results of their competitions. The HRV (mean heart rate, SDNN, RMSSD, TP, LF, HF, LF/HF ratio) was measured in the resting status. A t-test was used to compare the two groups, and bivariate logistic regression analysis was performed to determine the HRV elements that affect performance. The data were analyzed using IBM SPSS Statistics ver. 24.0 (IBM Co., Armonk, NY, USA).

RESULTS: The mean heart rate was higher in the high performance group (72.88) than in the low performance group

†Corresponding Author : Hyun-Chul Kim

icandox77@kyungnam.ac.kr, https://orcid.org/0000-0002-9154-565X

This is an Open Access article distributed under the terms of the Creative Commons Attribution Non-Commercial License (http://creativecommons.org/licenses/by-nc/3.0) which permits unrestricted non-commercial use, distribution, and reproduction in any medium, provided the original work is properly cited.
(64.75) $(\mathrm{p}=.049)$. The $\mathrm{LF} / \mathrm{HF}$ ratio was higher in the high performance group (3.43) than in the low performance group (0.83), and the results were significant $(\mathrm{p}=.038)$. No HRV elements having a significant effect on the performance were observed.

CONCLUSION: This study showed that the activity of the sympathetic nervous system was dominant in the high performance group in the resting status than in the low performance. The high performance group is believed to be in the overtraining status who experience more stress.

Key Words: Heart rate, Autonomic nervous system, Judo, Athletes

\section{Introduction}

Elite athletes experience considerable physical and mental stress. They suffer physical stress because of the high intensity training and competition, and they experience mental stress from anxiety, tension, frustration with competition, and training.

This physical and mental stress affects the function of the autonomic nervous system and the heart rate [1,2]. If the stress and overtraining status continues, the function of the autonomic nervous system can break down, which 
will have adverse health effects. Therefore, athletes need to take proper rest so that the function of the autonomic nervous system can be well maintained.

The autonomic nervous system consists of the sympathetic and parasympathetic nervous systems. The sympathetic nervous system is called the "fight \& flight" response. This mainly causes excitement of the body, such as increasing the heart rate. The parasympathetic nervous system mainly causes the recovery response, such as decreasing heart rate. In addition, it also enhances digestion by stimulating salivary gland secretion. Therefore it is called the "rest \& digest" response [3].

The function of the autonomic nervous system can be measured through heart rate variability. The heart rate variability means subtle changes in heart rate [4]. The heart rate changes constantly under the control of the sympathetic and parasympathetic nervous system in response to changes to the environment, stress, and respiration [5]. Therefore, the function and balance of the autonomic nervous systems can be evaluated by measuring the heart rate variability.

The heart rate variability is used medically to predict arrhythmia after myocardial infarction, sudden cardiac death, and diabetic neuropathy [6,7]. A previous study reported that after experiencing a myocardial infarction, patients with low heart rate variability had lower survival rates than those with higher heart rate variability [8].

The heart rate variability is also used to monitor an athlete's training program, overtraining syndrome, or exercise performance in sports $[9,10]$. Research on the use of heart rate variability in the sports area has been conducted.

According to a previous study about on the heart rate variability of young archery athletes, experienced athletes had higher LF and RMSSD values during competition than beginners. This means that the parasympathetic nervous system of experienced archers is more dominant than beginners [11]. In another study on the effects of heart rate variability on the performance of elite shooting athletes, the high performance group had a lower SDNN than the low performance group [12]. High performance athletes experience more mental stress because they used more attention and cognitive ability than low performance athletes. This stressful situation caused a decrease in the SDNN of the high performance groups.

Several studies have examined the effects of the heart rate variability of archery and shooting athletes. On the other hand, there has been little research on measurements of the heart rate variability of the combat sports athletes in South Korea.

Combat sports athletes repeat high-intensity training and experience considerable stress from losing weight before the match. This could have negative effects on the autonomic nervous function of combat sports athletes. Therefore, this study investigated the autonomic nervous function of elite female judo athletes by measuring the Heart rate variability. In addition, the difference in the autonomic nervous function according to their performance was also investigated.

\section{METHODS}

\section{Participants}

Sixteen elite female judo athletes training at the Korean National Training Center in 2017 participated in this study. The average age of the subjects was 24.8 years. The athletes who won medals of the international competition, such as the Olympics, Asian Games, and World Championships over the last three years, were classified as high performance groups. Those who had not won medals over the same time period were classified as low performance groups.

\section{Ethics approval and consent to participate}

The study was performed according to the Declaration of Helsinki. The personal information of the participants was not revealed. The heart rate variability measurements 
were non-invasive, and simple contact measurement equipment was used. The measurements did not cause any changes to the subjects' physical condition. Each subject was fully informed of the purpose of the study and agreed to participate in this study.

\section{Heart Rate Variability measurement}

The heart rate variability was measured to investigate the autonomic nervous function of the participants. The participants were prohibited from smoking, drinking, consuming drugs, or beverages that could stimulate the autonomic nervous system from the day before the measurement. The measurement was performed at 3:00 pm at least two hours after a meal. The participants were allowed to rest in the laboratory for 30 minutes before the measurement and the measurements were taken in a stable status. The measurements were taken for three minutes.

The participants were asked to breathe normally without moving or talking during the measurements. The measurement equipment was SA-6000, MEDICO Co., Ltd., Korea.

\section{1) Time domain analysis [13]}

Time domain analysis of the heart rate variability was based on the $\mathrm{NN}$ (normal to normal) interval in continuous ECG measurements. Time domain analysis consisted of the mean heart rate, SDNN, and RMSSD. The SDNN (Standard Deviation of the NN Interval) is the standard deviation of the NN interval during the test [13], and was calculated as the square root of the variance of $\mathrm{NN}$ interval. This indicates a change in the heartbeat during the recording time. The SDNN reflects the total variability $[13,14]$. The reduction of the SDNN reflects the reduced ability of the autonomic nervous system to control and cope with stress. The RMSSD (Root Mean Square of Successive Differences) is calculated as the square root of the mean of squared differences between the adjacent RR intervals
[13]. This is a commonly used variable when evaluating the activity of the parasympathetic nerves associated with the cardiovascular system $[13,14]$.

\section{2) Frequency domain analysis [13]}

The activity of the sympathetic and parasympathetic nerves can be analyzed by frequency domain analysis of the heart rate variability. Total power (TP) reflects the overall activity of the autonomic nervous system, such as the SDNN. The decrease in total power reflects a reduction in the autonomic nervous system activity [13]. A low frequency (LF) means measurement of the power in the low-frequency range $(0.04-0.15 \mathrm{~Hz})$. This reflects the activity of the sympathetic nervous system and parasympathetic nervous system, but it is used mainly as an index of the sympathetic nervous system activity [13]. A high frequency (HF) indicates the power in the high-frequency range $(0.15-0.4 \mathrm{~Hz})$. This is regulated by the parasympathetic nervous system (vagus nerve) and reflects the activity of the parasympathetic nervous system [13]. The LF/HF ratio indicates the ratio of sympathetic and parasympathetic nervous system activity. A high LF/HF ratio indicates the dominance of sympathetic nervous system activity [13].

\section{Data analysis}

The data were analyzed using IBM SPSS Statistics ver. 24.0 (IBM Co., Armonk, NY, USA). A t-test was performed to determine the differences in the heart rate variability between the high performance and low performance groups. In addition, bivariate logistic regression analysis was performed to identify the HRV elements that affect the performance. A $\mathrm{p}$ value $<.05$ was considered significant.

\section{RESULTS}

The participants were classified into a high performance 
Table 1. General Characteristics of the Participants

\begin{tabular}{ccccc}
\hline Group & Number & Age (mean, years) & Height (mean, cm) & Weight $(\mathrm{mean}, \mathrm{kg})$ \\
\hline Low Performance & 8 & 23.00 & 164.75 & 63.63 \\
High Performance & 8 & 25.75 & 167.25 & 68.75 \\
Total & 16 & 24.38 & 166.00 & 66.19 \\
\hline
\end{tabular}

High Performance: Athletes won medals at international games in the past three years

Low Performance: Athletes had not won medals in international games in the past three years

Table 2. Difference of HRV According to Performance (Time Domain Analysis)

\begin{tabular}{|c|c|c|c|c|c|c|}
\hline Variation & Group & Value & SD & $\mathrm{F}$ & $\mathrm{t}$ & $\mathrm{P}$ \\
\hline \multirow{2}{*}{$\mathrm{HR}$} & Low Performance & 64.75 (times) & 5.75 & \multirow{2}{*}{1.728} & \multirow{2}{*}{-2.152} & \multirow{2}{*}{$.049^{*}$} \\
\hline & High Performance & 72.88 (times) & 8.99 & & & \\
\hline \multirow{2}{*}{ SDNN } & Low Performance & $60.32(\mathrm{~ms})$ & 13.21 & \multirow{2}{*}{.454} & \multirow{2}{*}{1.112} & \multirow{2}{*}{.285} \\
\hline & High Performance & $52.18(\mathrm{~ms})$ & 15.97 & & & \\
\hline \multirow{2}{*}{ RMSSD } & Low Performance & $57.35(\mathrm{~ms})$ & 19.18 & \multirow{2}{*}{.721} & \multirow{2}{*}{1.680} & \multirow{2}{*}{.115} \\
\hline & High Performance & $40.35(\mathrm{~ms})$ & 21.25 & & & \\
\hline
\end{tabular}

$*_{\mathrm{p}}<.05$

HRV : Heart rate variability

High Performance: Athletes won medals at international games in the past three years

Low Performance: Athletes had not won medals in international games in the past three years

group (8) and a low performance group (8) according to their performance. Table 1 lists the general characteristics of the participants.

The mean heart rate was higher in the high performance group (72.88) than in the low performance group (64.75), and the data were statistically significant $(p=.049)$. The SDNN and RMSSD were higher in the low performance group than in the high performance group but the data were not statistically significant (Table ${ }^{*} p<.052$ ).

The total power (TP) and low frequency (LF) did not show a significant difference between the two groups. The high frequency (HF) was higher in the low performance group (6.42) than in the high performance group (5.50) but the difference was not statistically significant $(\mathrm{p}=.170)$. The LF/HF ratio was significantly $(p=.038)$ higher in the high performance group (3.43) than in the low performance group (0.83) (table 3 ). This means that the activity of the sympathetic nerves was higher in the high performance group than in the low performance group.

Bivariate logistic regression analysis was performed to determine the heart rate variability factors affecting performance. On the other hand, this study did not find any heart rate variability elements that had a significant effect on performance (table 4).

\section{DISCUSSION}

In this study, the heart rate variability was measured to investigate the relationship between the autonomic nervous function and performance of national female judo players. The participants were divided into a high and low performance group based on their performance. The heart rate variability parameters (SDNN, RMSSD, TP, LF, HF, $\mathrm{LF} / \mathrm{HF}$ ) of the two groups were also compared. 
Table 3. Difference in the HRV According to Performance (Frequency Domain Analysis)

\begin{tabular}{|c|c|c|c|c|c|c|}
\hline Variation & Group & Value & SD & $\mathrm{F}$ & $\mathrm{t}$ & $\mathrm{P}$ \\
\hline \multirow{2}{*}{$\mathrm{TP}$} & Low Performance & 7.67(ms2) & 0.65 & \multirow{2}{*}{1.177} & \multirow{2}{*}{.317} & \multirow{2}{*}{.756} \\
\hline & High Performance & $7.55(\mathrm{~ms} 2)$ & 0.90 & & & \\
\hline \multirow{2}{*}{$\mathrm{LF}$} & Low Performance & $6.03(\mathrm{~ms} 2)$ & 1.02 & \multirow{2}{*}{.015} & \multirow{2}{*}{-.967} & \multirow{2}{*}{.350} \\
\hline & High Performance & $6.51(\mathrm{~ms} 2)$ & 0.97 & & & \\
\hline \multirow{2}{*}{$\mathrm{HF}$} & Low Performance & $6.42(\mathrm{~ms} 2)$ & 1.12 & \multirow{2}{*}{.714} & \multirow{2}{*}{1.446} & \multirow{2}{*}{.170} \\
\hline & High Performance & $5.50(\mathrm{~ms} 2)$ & 1.40 & & & \\
\hline \multirow{2}{*}{$\mathrm{LF} / \mathrm{HF}$} & Low Performance & 0.83 & 0.52 & \multirow{2}{*}{5.518} & \multirow{2}{*}{-2.521} & \multirow{2}{*}{$.038^{*}$} \\
\hline & High Performance & 3.43 & 2.87 & & & \\
\hline
\end{tabular}

$* \mathrm{p}<.05$

HRV : Heart rate variability

High Performance: Athletes won medals at international games in the past three years

Low Performance: Athletes had not won medals in international games in the past three years

Table 4. The HRV Factors That Associated with Performance

\begin{tabular}{cccc}
\hline Dependent Variable & Independent Variable & Exp(B) & P-value \\
\hline & Average HR & 1.037 & .759 \\
TP & LF & 2332.061 & .135 \\
Performance & HF & .156 & .381 \\
& LFHF & 5.351 & .556 \\
& SDNN & 3.392 & .124 \\
& RMSSD & .764 & .159 \\
\hline
\end{tabular}

HRV : Heart rate variability

The mean heart rate was higher in the high performance group (72.88) than in the low performance group (64.75) $(p=.049)$. The $\mathrm{LF} / \mathrm{HF}$ ratio was higher in the high performance group (3.43) than in the low performance group (0.83) and the difference was statistically significant $(p=.038)$. This means that the activity of the sympathetic nervous system is more dominant in the high performance group than in the low performance group. Activation of the sympathetic nervous system represents a state of excitement or stress. Therefore, the high performance group can be assumed to be more excited or stressed than the low performance group, but no heart rate variability elements that had a significant effect on performance were found.

A previous study on the relationship between the performance and heart rate variability of archery athletes showed that high-performance athletes had a high HF, low LF, and low LF/HF ratio [15]. In another study on archery athletes, more skilled athletes showed higher scores and lower heart rates at competition than less experienced athletes [16]. This means that archers with good performance have higher activity of the parasympathetic nerves than sympathetic nerves. The results of these previous studies were in contrast to the results of the present 
study, where the sympathetic nervous activity of the high performance group was higher than that of the low performance group.

Archery and judo have different characteristics. Archery is a record game, and it is played in a stable status. On the other hand, judo is a combat sport and victory is determined by fighting an opponent for five minutes. The activity of the sympathetic nervous system, which increases the heart rate and cardiac output, is believed to be more important in judo than archery. On the other hand, the heart rate variability was measured at rest, not during competition in this study. Therefore, it cannot be concluded that the characteristics of the judo performance influenced the dominance of the sympathetic nerves in the high performance group in this study. Therefore, further research on the heart rate variability measurement during exercise using wearable equipment is needed.

Measurements of the heart rate variability are sometimes used as an indicator of overtraining syndrome. Overtraining syndrome is a condition in which hormonal, biological, and neurological regulating mechanisms are disrupted continuously due to excessive training, and includes mood disorders [17].

In previous studies, players with overtraining syndrome showed a decreased SDNN, RMSSD, or a disproportionate change in the LF/HF ratio, such as an increased or decreased LF/HF ratio. A previous study compared the heart rate variability of seven overtraining groups, eight endurance training groups, and eight sedentary groups [18]. The overtraining group had a higher LF/HF ratio than the other groups. In another study of 15 female athletes, the overtraining group also reported an increase in the LF compared to the control group [19]. These results show that the activity of the sympathetic nervous system is predominant in overtraining syndrome.

In a previous study of judo players, the high training load group showed a lower RMSSD, HF level, higher Lf/HF ratio, and higher stress index than the moderate training load group [20]. This also means that the activity of the sympathetic nervous system is increased and the activity of the parasympathetic nerves is decreased in the high training load group. This suggests that heart rate variability measurements can be used to monitor stress and recovery in judo players.

In this study, the LF/HF ratio was higher in the high performance group than in the low performance group in the resting status. According to a previous study [18-19-20], it can be interpreted that the high performance group had overtrained. High performance athletes may be more stressed physically and psychologically because of the pressure of ranking and high intensity training than low performance athletes. This may result in overtraining syndrome in high performance athletes that can lead to a functional imbalance of the autonomic nervous system. On the other hand, this study did not analyze the intensity, amount of training and stress of athletes.

These results suggest that measurements of the heart rate variability can help monitor the overtraining status in elite athletes. In addition, if measurements of the heart rate variability are poor, some methods can be applied to improve it. The heart rate variability biofeedback breathing can improve the function of the autonomic nervous system $[21,22]$. In a case study of 14-year-old golfers, competition anxiety decreased and performance improved after ten sessions of breathing exercise with biofeedback of the heart rate variability [21]. Therefore, elite athletes with an autonomic dysfunction need to apply a heart rate variability biofeedback breathing method to relieve tension and improve the autonomic nervous system function.

This study had some limitations. First, the subjects were limited to elite female judo players. Therefore, the result cannot be generalized. In addition, the number of participants was low at 16 persons. On the other hand, this study focused on national elite athletes and combat sports athletes. Moreover, there have been few studies on heart rate variability of combat sports in domestic sports 
fields, which may explain the value of this study.

In this study, the heart rate variability was measured only at rest. Measuring the heart rate variability at competition or in training would have been better. On the other hand, high activity of the sympathetic nervous system in the resting state can mean stress and overtraining. The heart rate variability measurements during the resting state can identify the athletes' overtraining and stress conditions, which also explains the value of this study.

\section{CONCLUSION}

This study compared the heart rate variability between the high and low performance groups. The results showed that the high performance group had a significantly higher mean heart rate and $\mathrm{LF} / \mathrm{HF}$ ratio than the low performance group.

High performance group had more dominant sympathetic nervous activity than the low performance group. This suggests that the high performance group was more overtrained and stressed for competition than the low performance group. The heart rate variability can be improved through the heart rate variability biofeedback breathing.

Based on these results, further study will be needed to determine if measurements of the heart rate variability can predict the performance and overtraining syndrome of athletes. In addition, it is important to study training methods, such as biofeedback breathing, which can improve the autonomic nervous function of elite athletes.

\section{Acknowledgment}

All authors appreciate the players who cooperated with the measurements.

\section{References}

[1] McCraty R, Atkinson M, Tiller WA, et al. The effects of emotions on short-term powerspectrum analysis of heart-rate variability. Am J Cardiol. 1995;76(14):1089-93.

[2] Rimoldi O, Furlan R, Pagani MR, et al. Analysis of neuralmechanisms accompanying different intensities of dynamic exercise. Chest. 1992;101(5):226-30.

[3] Draper N, Hodgson C. Adventure Sport Physiology. Chennai, India: Wiley-Blackwell. 2008.

[4] Acharya UR, Joseph KP, Kannathal N, et al. Heart rate variability: A review. Med Biol EngComput. 2006; 44(12):1031-51.

[5] Dong JG. The role of heart rate variability in sports physiology. Exp Ther Med. 2006;11(5):1531-6.

[6] Khandoker AH, Jelinek HF, Palaniswami M. Identifying diabetic patients with cardiac autonomicneuropathy by heart rate complexity analysis. Biomed Eng Online. 2009;8(3).

[7] Tereshchenko LG, Cygankiewicz I, McNitt S, et al. Predictive value of beat to beat QT variability index across thecontinuum of left ventricular dysfunction: Competing risks of non cardiac or cardiovasculardeath and sudden or nonsudden cardiac death.Circ Arrhythm Electrophysiol. 2012;5(4):719-27.

[8] Wolf MM, Varigos GA, Hunt D, et al. Sinus arrhythmia in acute myocardial infarction.Med J Aust. 1978;2(2): 52-3.

[9] Makivić B, Djordjević NM, Willis MS. Heart Rate Variability(HRV) as a Tool for Diagnostic and MonitoringPerformance in Sport and Physical Activities. JEPonline. 2013;16(3):103-31

[10] Plews DJ, Laursen PB, Kilding AE, et al. Heart rate variability in elite triathletes, is variation in variability the key to effective training? A case comparison. Eur J Appl Physiol. 2012;112(11):3729-41.

[11] Carrillo AE, Christodoulou VX, Koutedakis Y, et al. Autonomic nervous systemmodulation during an archery 
competition in novice and experienced adolescent archers. JSports Sci. 2011;29(9):913-7

[12] Jon JH. The effect of resting heart rate variability on shooting performance among rapid fire pistolathletes. Exerc Sci. 2015;24(3):315-21.

[13] Task Force of The European Society of Cardiology and The North American Society of Pacing andElectrophysiology. Heart rate variability. Standards of measurement, physiological interpretation, andclinical use. Circulation. 1996;93(5):1043-65.

[14] Kleiger RE, Stein PK, Bigger JT Jr.Heart Rate Variability: Measurement and Clinical Utility. Ann Noninvasive Electrocardiol. 2005;10(1):88-101.

[15] Lo CT, Huang SH, Hung TM. A study of the relationship between heart rate variability and archeryperformance. Int J Psychophysiol. 2008;63(4):181-7.

[16] Clemente F, Couceiro M, Rocha R, et al. Study of the Heart Rate and AccuracyPerformance of Archers. Journal of Physical Education and Sport. 2011;11(4):434-7.

[17] Meeusen R, Duclos M, Foster C, et al. Prevention, diagnosis and treatment of the overtraining syndrome:
Joint consensusstatement of the European College of Sport Science and the American College of Sports Medicine.EUR J SPORT. 2013;13(1):1-24.

[18] Mourot L, Bouhaddi M, Perrey S, et al. Decreasein heart rate variability with overtraining: Assessment by the Poincare plot analysis. Clin PhysiolFunct Imaging.2004;24(1):10-8.

[19] Uusital ALT, Uusitalo A J, Rusko HK. Heart rate and blood pressure variability during heavy training an overtraining in the female athletes.Int $J$ Sports Med.2000;21(1):45-53.

[20] José M, Álamo JM, García-Massó X, et al. Use of Heart Rate Variability in Monitoring Stress and Recovery in Judo Athletes. J Strength Cond Res. 2014;28(7):1896-905

[21] Lagos L, Vaschillo E, Vaschillo B, et al. Heart Rate Variability Biofeedback asa Strategy for Dealing with Competitive Anxiety: A Case Study. Biofeedback. 2008;36(3):109-15.

[22] Lehrer P, Vaschillo B, Zucker T, et al. Protocol for HeartRate Variability Biofeedback Training. Biofeedback. 2013;41(3):98-109. 